\title{
Estudio comparativo del efecto de las asociaciones anestésicas atropina-tiletamina/ zolazepam y atropina-ketamina/diazepam en emúes (Dromaius novaehollandiae) adultos
}

\author{
Effects of the anaesthetic associations atropine-tiletamine/zolazepam and \\ atropine-ketamine/diazepam on adult emus (Dromaius novaehollandiae)

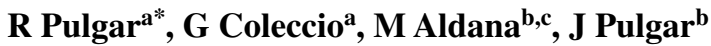 \\ ${ }^{a}$ Escuela de Medicina Veterinaria, Universidad Santo Tomás, Santiago, Chile.

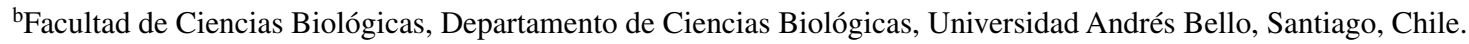 \\ 'Facultad de Ecología y Recursos Naturales, Universidad Andrés Bello, Santiago, Chile.
}

\section{SUMMARY}

\begin{abstract}
In this study, the effects of the anaesthetic associations atropine IM $(0.05 \mathrm{mg} / \mathrm{kg})$-tiletamine/zolazepam IV (4 mg/kg total) and atropine IM $(0.05 \mathrm{mg} /$ $\mathrm{kg})$-ketamine IV $(5 \mathrm{mg} / \mathrm{kg}$ total $) /$ diazepam $(0.5 \mathrm{mg} / \mathrm{kg})$ on physiological, anaesthetic and biochemical responses were determined on adult emus. Animals ( $\mathrm{n}=7$ per group) were randomly assigned to the two anaesthetic associations. Variables were measured at baseline, during surgical plane and during $30 \mathrm{~min}$ of observation. Heart rate and temperature increased at the beginning of the experimental period (between 5-10 min, $\mathrm{P}=0.001$ ), while respiratory frequency and pulse decreased (between 5-15 min, $\mathrm{P}=0.003$ ). These patterns were detected with both anaesthetic associations. Anaesthetic induction and recuperation time were not affected by treatments $(\mathrm{P}=0.12$ and $\mathrm{P}=0.13$ respectively). Emus treated with tiletamine showed a greater anaesthetic time than emus treated with ketamine $(\mathrm{P}=0.012)$. Significant increases in A.S.T. and glucose were observed at $24 \mathrm{~h}$ post application of anaesthetic associations, resulting higher in ketamine emus $(\mathrm{P}=0.006$ and $\mathrm{P}=0.008$ respectively). Finally, there were no significant differences between anaesthetic treatments for haemoglobin, total proteins and uric acid $(\mathrm{P}=0.99, \mathrm{P}=0.97$ and $\mathrm{P}=0.81$ respectively). In conclusion, atropine-tiletamine/ zolazepam and atropine-ketamine/diazepam protocols resulted safe and efficient for animal manipulation, however the greater anaesthetic time observed in tiletamine-treated animals may determine preference for this anaesthetic combination.
\end{abstract}

Palabras clave: emú, anestesia, tiletamina, ketamina.

Key words: emus, anaesthetic, tiletamine, ketamine.

\section{INTRODUCCIÓN}

La anestesia general puede ser definida como la acción de fármacos que suprimen de manera temporal la sensibilidad y la movilidad de un animal, sin afectar sus funciones vitales (Rang y col 2004). Un protocolo anestésico debe producir inconciencia, amnesia, analgesia y relajación muscular (López-Timoneda y Gasco 1993). Sin embargo, actualmente no existe un fármaco que incluya todas las características de analgesia e inconciencia requeridas (Laredo 2001), por lo que se recurre a la combinación de tranquilizantes, relajantes musculares, anestésicos y analgésicos.

Entre los anestésicos inyectables empleados en aves encontramos: barbitúricos (tiopental), fenotiacínicos (acepromacina), disociativos (ketamina, tiletamina-zolazepam), agonistas $\alpha_{2}$ adrenérgicos (xilazina) y propofol (2,6-di-isopropilfenol) (Curro 1998, Alves y col 2003). Entre los fármacos menos recomendables se encuentran los barbitúricos e hidrato de cloral (Cornick-Seahorn 1996). Para aves corredoras de gran

Aceptado: 25.02.2009.

* Ejército 146, Santiago, Chile; rpulgar@ust.cl tamaño, la anestesia inyectable es usada en procedimientos cortos, donde las combinaciones anestésicas incluyen: agonistas $\alpha_{2}$ adrenérgicos seguidos de ketamina o benzodiazepina seguida por ketamina, tiletamina-zolazepam (Van Heerden y Keffen 1991). Uno de los primeros estudios acerca del uso de anestésicos inyectables en emúes lo desarrollaron Grubb y col (1983), quienes determinaron que para realizar cualquier procedimiento quirúrgico se debe usar una dosis de ketamina de $25 \mathrm{mg} / \mathrm{kg}$ por vía intramuscular, asociado a una inyección endovenosa adicional de $5-8 \mathrm{mg} / \mathrm{kg}$. Sin embargo, se desconoce qué anestésico podría ser mejor tolerado por los emúes.

Dentro de los anestésicos disociativos aplicados en aves, la ketamina es el más usado en la actualidad (Muir y col 2001, Mostachio y col 2008). Se ha demostrado que la respuesta supresora de la ketamina es producto de la estimulación específica de la actividad nerviosa adrenérgica, sobre receptores $\alpha$, más que por el bloqueo del nervio vago (Sumano y Ocampo 1997). Otros sitios de acción de la ketamina incluyen el bloqueo de transporte neuronal de aminas neurotransmisoras como serotonina, dopamina, noradrenalina (Smith y col 1981). En adición, la ketamina participa en la inhibición de las áreas de formación reticular medular y potencia los efectos del GABA (ácido gamma aminobutírico) sobre el sistema nervioso central (SNC). 
El efecto analgésico principal de la ketamina se atribuye a su acción sobre los receptores NMDA (N-Metil-Daspartato) en el núcleo del tracto solitario y en menor medida a estimulación de los receptores opiáceos mu y sigma del cerebro y la médula espinal (Miller 2001). La ketamina actúa sobre los receptores NMDA inhibiendo su activación por glutamato, neurotransmisor que excita el SNC (Kohrs y Durieux 1998). Finalmente, la acción sobre los receptores NMDA podría mediar el mecanismo de estimulación simpática central.

La ketamina se distribuye rápidamente en todos los tejidos del organismo, principalmente adiposo, hígado, pulmones y encéfalo de pequeños animales y aves (Adams 1995, Paddleford 2001, De Lucas y col 2007). Este fármaco se utiliza comúnmente en combinación con benzodiazepinas o con agonistas $\alpha_{2}$ adrenérgicos para mejorar la relajación y la profundidad de la anestesia en aves (Van Heerden y Keffen 1991). En general, luego de la administración de ketamina, es posible observar variaciones de la temperatura corporal, aumento del gasto cardiaco y presión arterial (Sumano y Ocampo 1997, Paddleford 2001).

Otro anestésico disociativo es la tiletamina, fármaco relacionado tanto química como estructuralmente con la ketamina ya que ambos son derivados de la fenciclidina (Adams 1995). Sin embargo, la tiletamina posee una mayor potencia anestésica y presenta efectos secundarios tales como rigidez muscular y convulsiones tónico-clónicas intensas y de larga duración. La inducción anestésica con tiletamina es rápida, aunque se han registrado recuperaciones violentas si es administrado por vía endovenosa (Cornick y Jensen 1992, Lin y col 1997). Al igual que la ketamina, la tiletamina se administra asociada a una benzodiazepina como el zolazepam, lo que suaviza la recuperación anestésica (Lin y col 1997). El mecanismo de acción de la tiletamina es similar al de la ketamina. La mayor desventaja parece ser que a altas dosis provoca una anestesia de corta duración seguida de una larga (2-4 horas) y a veces difícil recuperación (Cornick y Jensen 1992).

El emú (Dromiceidae: Dromaius novaehollandiae) es un ave corredora del orden de los Casuariformes, originaria de las grandes planicies australianas. Pertenece al grupo de las Ratites, el que incluye a todas las aves terrestres, al igual que el avestruz, ñandú, el kiwi y los casuarios (Stewart 1994, Johnson 1996). Anatómicamente, el emú es más pequeño que el avestruz, puede llegar a medir 1,5 metros de altura con un peso superior a los $36 \mathrm{~kg}$ el macho, mientras que la hembra alcanza 1,8 metros y $55 \mathrm{~kg}$ (Ritchie 1994, Tully y Shane 1996). Al nacer, el emú es de color negro oscuro, sin embargo, a medida que va creciendo toma un aspecto jaspeado, lo que le permite mimetizarse muy bien entre la vegetación (Perrin y Middleton 1990).

En este contexto, el objetivo del presente trabajo fue comparar dos protocolos anestésicos disociativos disponibles en el mercado nacional: Atropina Tiletamina-Zolazepam y Atropina Ketamina-Diazepam en emúes adultos.

\section{MATERIAL Y MÉTODOS}

Se seleccionaron 14 ejemplares de emúes adultos, de dos años de edad cada uno y de un peso promedio de $33 \pm 4 \mathrm{~kg}$. Las aves se distribuyeron aleatoriamente en dos grupos de 7 animales cada uno. Para evitar la regurgitación del alimento, previo a los ensayos, todas las aves fueron mantenidas en ayuno durante 12 horas y posteriormente se aplicaron los protocolos experimentales.

Previo a la toma de muestras y registro de observaciones en ambos grupos, se realizó un examen (EP) que consistió en evaluar el peso corporal (incluida condición corporal en una escala de 1 a 5), las mucosas (color, humedad, presencia o ausencia de hemorragias), la temperatura corporal y la frecuencia cardiaca y respiratoria. Luego a cada uno de los animales experimentales se le aplicó la premedicación descrita en el cuadro 1; al cabo de 15 min se realizó la inducción anestésica. Posteriormente, se constató que el animal estuviera en plano quirúrgico (PQ), que fue definido como la ausencia de reacción frente al dolor provocado por la aplicación de una pinza Backhaus, en los cojinetes plantares y directamente sobre las falanges del ave. Todos los animales fueron mantenidos sobre una superficie de espuma de poliuretano, y cada 5 minutos se registraron las siguientes variables:

Cuadro 1. Protocolos anestésicos utilizados en los emúes.

Anaesthetic protocols used in emus.

\begin{tabular}{|c|c|c|c|c|c|c|}
\hline & \multicolumn{3}{|c|}{ GRUPO A (7 emúes) } & \multicolumn{3}{|c|}{ GRUPO B (7 emúes) } \\
\hline & Fármacos & Dosis & Vía & Fármacos & Dosis & Vía \\
\hline Premedicación & Atropina & $0,05 \mathrm{mg} / \mathrm{kg}$ & IM & Atropina & $0,05 \mathrm{mg} / \mathrm{kg}$ & IM \\
\hline Inducción & Tiletamina/Zolazepam & $4 \mathrm{mg} / \mathrm{kg}$ & $\mathrm{EV}$ & $\begin{array}{l}\text { Ketamina } \\
\text { Diazepam }\end{array}$ & $\begin{array}{c}5 \mathrm{mg} / \mathrm{kg} \\
0,5 \mathrm{mg} / \mathrm{kg}\end{array}$ & $\mathrm{EV}$ \\
\hline Mantención & \multicolumn{3}{|c|}{ Dosis efecto por 30 minutos. } & \multicolumn{3}{|c|}{ Dosis efecto por 30 minutos. } \\
\hline
\end{tabular}

$\mathrm{IM}=$ intramuscular, $\mathrm{EV}=$ endovenosa 
VARIABLES FISIOLÓGICAS

Frecuencia cardiaca (latidos/min): estimada mediante el uso de un fonendoscopio (Littman $\left.{ }^{\circledR}\right)$ aplicado en la zona ventrocraneal del hemitórax izquierdo.

Frecuencia respiratoria (ciclos/min): evaluada mediante observación directa de la distensión de la caja torácica en la zona costal.

Temperatura corporal $\left({ }^{\circ} \mathrm{C}\right)$ : registrada mediante el uso de un termómetro clínico digital, aplicado en la cloaca del animal.

Saturación de oxígeno (\% $\mathrm{SpO}_{2}$ ) y pulso (pulsaciones/ min): estimadas mediante el uso de un oxímetro de pulso (Medical Armstrong $®$ modelo AD-1000), el cual registra simultáneamente la saturación de oxígeno y el pulso del animal. El sensor fue aplicado en la lengua del emú.

\section{VARIABLES ANESTESIOLÓGICAS}

Estas variables corresponden a tiempos asociados a la acción de los distintos tratamientos anestesiológicos utilizados. Todos los tiempos fueron determinados mediante el uso de un cronómetro digital:

Tiempo de inducción (seg): corresponde al período de tiempo transcurrido desde la aplicación del anestésico hasta que el ave pierde la conciencia y cae decúbito esternal o lateral.

Tiempo de anestesia quirúrgica con la dosis de inducción ( $\mathrm{min}$ ): corresponde al periodo de tiempo transcurrido desde que el ave pierde el conocimiento hasta que manifiesta alguna forma de movimiento.

Tiempo de recuperación ( $\mathrm{min}$ ): corresponde al periodo de tiempo transcurrido desde la aplicación de la última dosis de anestésico hasta que el animal se colocó en decúbito esternal (cuadro 1).

\section{VARIABLES BIOQUÍMICAS}

Se obtuvieron muestras de sangre pertenecientes a ambos grupos en tres momentos distintos durante el procedimiento anestésico: antes de iniciar el procedimiento, al momento que se observó alguna forma de movimiento producto de la recuperación anestésica y a las 24 horas de finalizado el procedimiento. Para la toma de muestras se desinfectó el área yugular derecha con alcohol desnaturalizado al $96^{\circ}$ y posteriormente se realizó la venopunción con una jeringa de $10 \mathrm{ml}$ provista de aguja $\mathrm{n}^{\circ} 21 \mathrm{G}$. Por cada animal y momento de extracción, las muestras de sangre obtenidas fueron depositadas en dos tubos, uno sin aditivo, para evaluar aspartato aminotransferasa (AST), ácido úrico y glucosa, la que fue determinada inmediatamente poscolección. El segundo tubo con heparina se utilizó para determinar volumen globular aglomerado (V.G.A.), hemoglobina y proteínas totales.

El procedimiento en laboratorio para las variables en estudio fue el siguiente:
Volumen globular aglomerado V. G. A. (\%): la muestra de sangre fue centrifugada a $10.000 \mathrm{rpm}$ por $5 \mathrm{~min}$ y se determinó la proporción celular sobre el total de volumen contenido en el microtubo.

Hemoglobina ( $\mathrm{Hb} \mathrm{g} / \mathrm{dL}$ ): $0,02 \mathrm{ml}$ de sangre con anticoagulante (heparina) se homogeneizaron con $5 \mathrm{ml}$ de solución Drabkin a temperatura ambiente $\left(25^{\circ} \mathrm{C}\right)$ por diez minutos. Posteriormente se determinó la absorbancia por medio de un espectrofotómetro (Metertek® Digital SP 830) a una longitud de onda de $540 \mu \mathrm{m}$.

$A S T(U I / L)$, ácido úrico $(\mathrm{mg} / \mathrm{dL})$ y glucosa $(\mathrm{mg} / \mathrm{dL})$ : la determinación de estas variables fue realizada a partir de suero sanguíneo. Para esto se procedió a centrifugar la muestra a 3.500 rpm durante 10 minutos usando reactivos comerciales (Human $\left.{ }^{\circledR}\right)$. Posteriormente se utilizó un método de análisis enzimático colorimétrico (Microlab 200 Merck).

Proteínas totales $(\mathrm{g} / \mathrm{dL})$ : las proteínas totales fueron cuantificadas a partir de plasma sanguíneo proveniente de la determinación del hematocrito. Para esto se utilizó refractómetro óptico (Atago®, modelo T2-NE).

\section{ANÁLISIS ESTADÍSTICO}

El análisis estadístico fue realizado sobre el total de animales en estudio (por grupo). Para evaluar la magnitud de las diferencias de las variables fisiológicas, anestesiológicas y bioquímicas entre protocolos de anestesia y tiempos de observación, se utilizaron Modelos Lineales Generalizados (GLM) que incluyeron medidas repetidas (Zar 1996). La comparación específica entre los niveles del factor anestésico y el factor tiempo de observación se realizó a posteriori mediante la prueba de Tukey. Cuando las variables estaban expresadas en porcentaje $\left(\% \mathrm{O}_{2}\right.$ y \% V.G.A.), se aplicó la transformación arcoseno (arcoseno $\sqrt{\text { variable) }}$ para aproximar a una distribución normal (Zar 1996). A través de todo el estudio se utilizó un valor de significancia de $\mathrm{P}<0,05$ y todos los análisis fueron realizados con el software Statistica 6.0 (Statsoft. Inc 1995 Tulsa, USA).

\section{RESULTADOS}

\section{VARIABLES FISIOLÓGICAS}

La evaluación de la frecuencia cardiaca indicó que, independiente del anestésico utilizado, los animales de ambos tratamientos sufrieron un incremento del ritmo cardiaco a los cinco minutos posterior a la inducción anestésica, para luego disminuir hasta alcanzar su condición basal $(\mathrm{P}=0,001$, figura 1). Por otra parte, la temperatura corporal presentó un marcado incremento a partir de los 10 minutos en los animales de ambos tratamientos anestesiológicos $(\mathrm{P}=0,0001$, figura 2). La frecuencia respiratoria y el pulso disminuyeron a partir de la inducción anestésica, estabilizándose desde los $5 \mathrm{~min}$ para la frecuencia respiratoria y desde los 15 min para el pulso $(\mathrm{P}=0,003$, figuras 3 
y 4). Finalmente la saturación de oxígeno no presenta variaciones significativas ni entre tratamientos ni entre tiempos de observación $(\mathrm{P}=0,77)$.

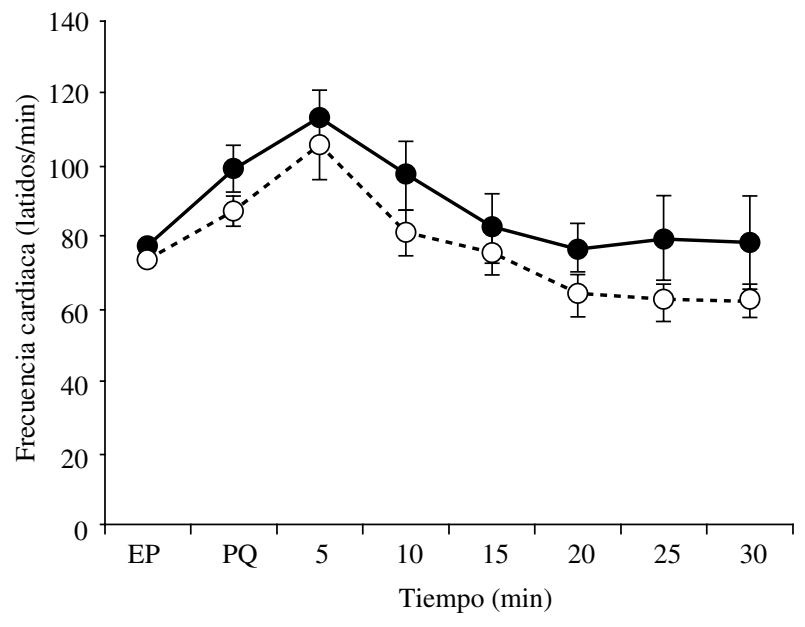

Tiletamina/zolazepam -O- Ketamina/diazepam

Figura 1. Frecuencia cardiaca de emúes expuestos a dos protocolos anestésicos, durante el tiempo experimental $(\mathrm{EP}=$ examen previo, $\mathrm{PQ}=$ plano quirúrgico). Las barras indican $+/-1$ error estándar.

Heart rate of emus exposed to two anaesthetic protocols, during experimental time $(\mathrm{EP}=$ Previous examination, $\mathrm{SP}=$ surgical plane). Bars indicate $+/-1$ standard error.

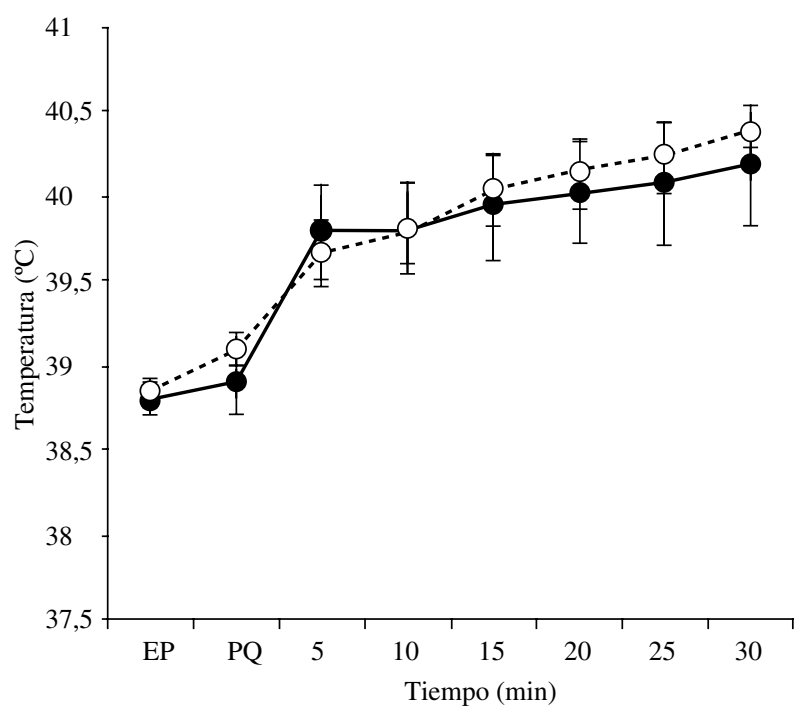

Tiletamina/zolazepam - o- Ketamina/diazepam

Figura 2. Temperatura de emúes expuestos a dos protocolos anestésicos, durante el tiempo experimental $(\mathrm{EP}=$ Examen preliminar, $\mathrm{PQ}=$ plano quirúrgico). Las barras indican $+/-1$ error estándar.

Temperature of emus exposed to two anaesthetic protocols during experimental time $(\mathrm{EP}=$ Previous to evaluation, $\mathrm{SP}=$ surgical plane). Bars indicate $+/-1$ standard error.

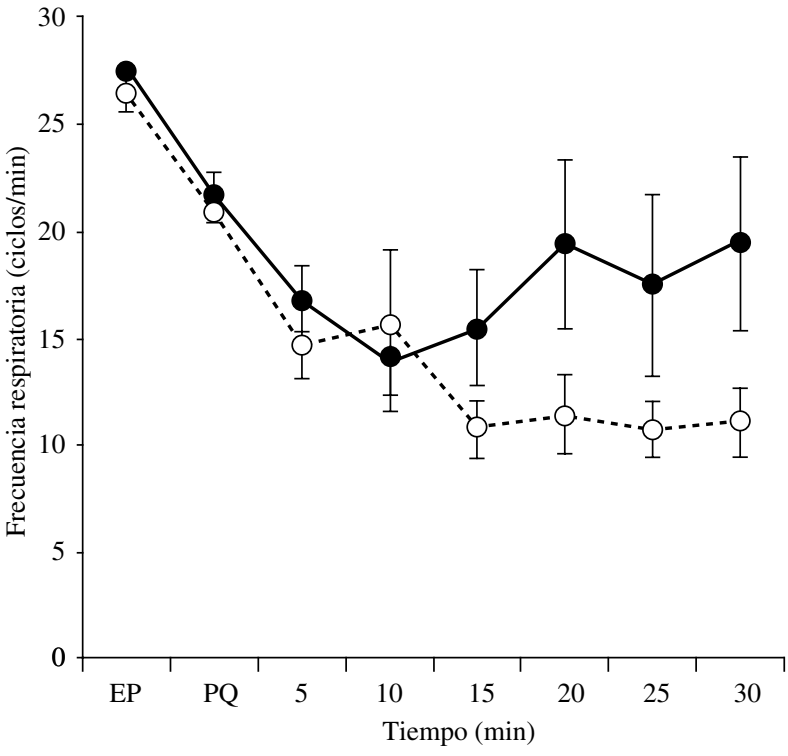

Tiletamina/zolazepam - o-Ketamina/diazepam

Figura 3. Frecuencia respiratoria de emúes expuestos a dos protocolos anestésicos, durante el tiempo experimental $(\mathrm{EP}=$ examen previo, $\mathrm{PQ}=$ plano quirúrgico). Las barras indican $+/-1$ error estándar.

Respiratory rate of emus exposed to two anaesthetic protocols during experimental time $(\mathrm{EP}=$ Previous examination, $\mathrm{SP}=$ surgical plane). Bars indicate $+/-1$ standard error.

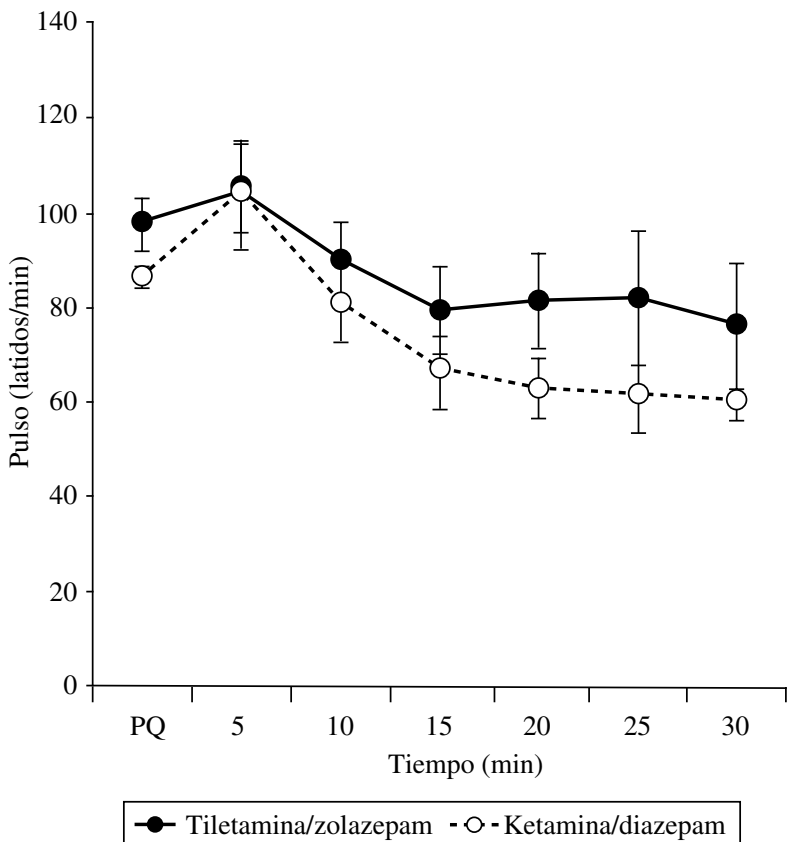

Figura 4. Pulso de emúes expuestos a dos protocolos anestésicos, durante el tiempo experimental $(\mathrm{EP}=$ examen previo, $\mathrm{PQ}=$ plano quirúrgico). Las barras indican +/- 1 error estándar.

Pulse of emus exposed to two anaesthetic protocols, during experimental time $(\mathrm{SP}=$ surgical plane $)$. Bars indicate $+/-1$ standard error. 


\section{VARIABLES ANESTESIOLÓGICAS}

La evaluación de las variables anestesiológicas indicó que los animales tratados con tiletamina presentaron un tiempo de anestesia quirúrgica mayor que aquellos tratados con ketamina ( $\mathrm{P}=0,012$, figura 5). Por otra parte, no se observaron diferencias al evaluar el tiempo de inducción y de recuperación entre protocolos anestésicos (Tiempo inducción $\mathrm{P}=0,13$, Tiempo recuperación $\mathrm{P}=0,12)$.

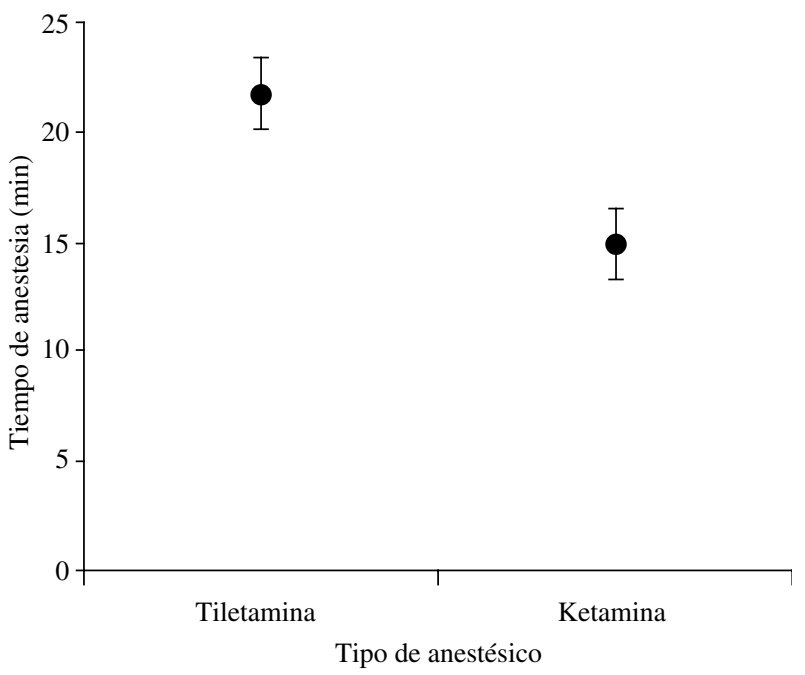

Figura 5. Tiempo de anestesia de emúes entre tratamientos. Las barras indican +/- 1 error estándar.

Anaesthesic time among emus treated with two different anaesthetics. Bars indicate +/- 1 standard error.

\section{VARIABLES BIOQUÍMICAS}

La evaluación de la A.S.T. reveló que independiente del tipo de anestesia utilizada se observó un aumento a las $24 \mathrm{~h}$ desde el término de su aplicación $(\mathrm{P}=0,006$, cuadro 2). Por otra parte, la cantidad de hemoglobina, proteínas totales y ácido úrico fue similar entre anestésicos y tiempos de observación $(\mathrm{P}=0,99, \mathrm{P}=0,97$, $\mathrm{P}=0,81$, respectivamente). Finalmente, al evaluar la concentración de glucosa entre anestésicos durante el tiempo de observación, los resultados indicaron que la glucosa resultó mayor en animales tratados con ketamina ( $P=0,003$, cuadro 3$)$. Sin embargo, en ambos tratamientos aumentó después de la aplicación del anestésico $(\mathrm{P}=0,008$, cuadro 3$)$.

\section{DISCUSIÓN}

Las combinaciones tiletamina-zolazepam y ketaminadiazepam producen anestesia de forma disociativa, por lo que tienden a aumentar el ritmo cardiaco y la presión arterial durante la fase de inducción, estimulando de forma
Cuadro 2. A.S.T. de emúes antes, durante y después de la aplicación de dos protocolos anestésicos. (e.s.) = error estándar.

A.S.T. of emus before, during and after the application of two anaesthetic protocols (e.s.) $=$ standard error.

\begin{tabular}{lcc}
\hline \multirow{2}{*}{$\begin{array}{c}\text { Momento } \\
\text { de la } \\
\text { observación }\end{array}$} & \begin{tabular}{c} 
Tratamiento \\
\cline { 2 - 3 } tiletamina- zolazepam
\end{tabular} & $\begin{array}{c}\text { Atropina } \\
\text { ketamina- diazepam }\end{array}$ \\
\hline Antes & $193,28(15,17)$ & $184,00(17,95)$ \\
Durante & $168,71(17,08)$ & $216,80(20,21)$ \\
Después & $331,28(37,01)$ & $310,80(43,79)$ \\
\hline
\end{tabular}

Cuadro 3. Niveles de glucosa de emúes antes, durante y después de la aplicación de dos protocolos anestésicos. (e.s.) = error estándar.

Blood glucose levels of emus before, during and after the application of two anaesthetic protocols. (e.s.) = standard error.

\begin{tabular}{lcc}
\hline \multirow{2}{*}{$\begin{array}{c}\text { Momento } \\
\text { de la } \\
\text { observación }\end{array}$} & \begin{tabular}{c} 
Tratamiento \\
\cline { 2 - 3 } tiletamina- zolazepam
\end{tabular} & $\begin{array}{c}\text { Atropina } \\
\text { ketamina- diazepam }\end{array}$ \\
\hline Antes & $133,85(12,98)$ & $183,83(14,02)$ \\
Durante & $157,57(18,03)$ & $178,50(19,48)$ \\
Después & $186,00(11,78)$ & $235,33(12,72)$ \\
\hline
\end{tabular}

adrenérgica el sistema cardiovascular (Adams 1995). Esta evidencia coincide con lo obtenido en el presente estudio; en ambos protocolos anestésicos los animales incrementaron su frecuencia cardiaca y pulso hasta los $5 \mathrm{~min}$ de anestesia, para luego disminuir a valores cercanos a los basales, lo que demuestra la proporcionalidad entre ambas variables fisiológicas (figuras 1 y 4, Guyton y Hall 1996, Pereira y col 1996). El incremento de la frecuencia cardiaca en nuestro estudio estaría asociado además al uso de atropina como premedicación en ambos protocolos, fármaco que es antagonista de los efectos de la acetilcolina en el nodo sinusal.

El empleo tanto de tiletamina-zolazepam como el de ketamina-diazepam tiende a aumentar la frecuencia respiratoria (Lin y col 1997), ya que generan una disminución del inotropismo y gasto cardiaco, alterando el intercambio de gases a nivel alveolar. La fuerte depresión de la frecuencia respiratoria observada en nuestro estudio (figura 3) estaría asociada al uso de dosis de anestésico mayores a las reportadas en la literatura para mamíferos (Tully y Shane 1996, Bruce 1998). El uso de altas dosis de anestesia en este estudio se fundamenta en que en las aves se encuentra el sistema portorrenal, el cual participa en procesos de detoxificación. Sin embargo, a pesar de las dosis utilizadas (cuadro 1), ninguno de los emúes experimentales sufrió de paro respiratorio o apnea transitoria. 
Lo anterior valida el procedimiento, ya que se considera que la apnea frecuentemente es el primer indicio de que se requiere intervención, producto de una excesiva profundidad anestésica o de complicaciones fisiológicas que pueden llevar a la muerte (Nevarez 2005).

La temperatura corporal tiende a descender de manera natural en individuos anestesiados debido a la disminución de la actividad muscular y al efecto que tienen las benzodiazepinas (diazepam y zolazepam) sobre el centro termorregulador. Lo anterior genera un comportamiento de poiquilotermia durante la fase anestésica, para luego recuperar su condición homeotérmica normal (Lin y col 1997, Sumano y Ocampo 1997, Paddleford 2001). En este estudio, contrario a lo esperado, se observó un leve incremento en la temperatura corporal de los emúes estudiados en ambos tratamientos (figura 2). Esta respuesta podría estar asociada a las condiciones ambientales de verano (aproximadamente $30^{\circ} \mathrm{C}$ ) bajo las cuales se realizó el trabajo de campo. En este sentido, se ha encontrado que la inmovilización de avestruces asociada a temperaturas ambientales elevadas disminuiría la frecuencia respiratoria generando una menor pérdida de calor desde la tráquea, sacos aéreos y faringe, y por ende incrementos en la temperatura cloacal de los avestruces (Silva de Carvalho y col 2007).

Los tiempos de inducción (tiletamina $50 \pm 13,54 \mathrm{~s}$ y ketamina $39,29 \pm 11,34 \mathrm{~s}$ ) y recuperación (tiletamina $35 \pm 12$ min y ketamina $48 \pm 16 \mathrm{~min}$ ) observados en el presente estudio son lo suficientemente breves para mantener la seguridad de los operadores y del animal. Estos resultados coinciden con los tiempos anestésicos reportados al usar tiletamina-zolazepam por vía intramuscular en avestruces (Silva de Carvalho y col 2007) y por vía intravenosa en emúes (Lin y col 1997). En avestruces, Ciboto y col (2006) establecieron un mayor tiempo de anestesia al usar tiletamina-zolazepam versus ketamina-diazepam, esto dado por la menor vida media de estos últimos. Este resultado concuerda con lo establecido por Adams (1995) en monos (Macaca facicularis) tratados con ketamina versus tiletamina-zolazepam. Estos antecedentes demostrarían una mayor potencia de tiletamina sobre ketamina, resultados que podrían ser determinantes al momento de realizar una elección entre estas dos asociaciones anestésicas.

La enzima A.S.T. no superó el valor máximo de referencia (380 UI/L) descrito por Fudge (1996). Sin embargo, en nuestro estudio se registró un aumento de esta enzima luego de 24 horas postaplicación de los anestésicos (cuadro 2). Este incremento en A.S.T. estaría asociado al trauma muscular de la inyección de atropina (premedicación) y traumatismos musculares generados por la excitación y delirio inherente al uso de agentes anestésicos disociativos (Lumeij 1997) durante la recuperación (Gunkel y Lafortune 2005).

La similitud en las cantidades de hemoglobina y proteínas totales entre animales tratados con ambos anestésicos indica que las aves no presentaban cuadros patológicos infecciosos de tipo crónico, procesos inflamatorios, estados de deshidratación, cuadros de desnutrición, anorexia, mala absorción ni falla hepática (Tully y Shane 1996). Por otra parte, las concentraciones de ácido úrico se mantuvieron dentro de los valores referenciales (1-13,3 mg/dl) descritos por Fudge (1996), lo que indicaría que en animales clínicamente sanos ambos protocolos resultan seguros para la funcionalidad renal. Finalmente la mayor cantidad de glucosa en los animales tratados con ketamina-diazepam (cuadro 3) estaría asociada al estrés sufrido por este grupo previo a la condición experimental, lo que habría provocado hiperglicemia (Stewart 1989). Para disminuir los niveles de estrés, se aumentó la dosis de diazepam, logrando una sedación y recuperación adecuada.

En resumen, los resultados obtenidos del presente estudio indican que ambos protocolos anestésicos se comportan de manera similar, por lo que serían igualmente útiles para inmovilizar a los emúes y realizar procedimientos de campo. Sin embargo, el mayor tiempo de anestesia quirúrgica observado en animales tratados con tiletamina (figura 5) podría determinar una preferencia por este fármaco versus la ketamina. Finalmente, el presente trabajo representa un avance significativo en el estudio de la anestesiología en Ratites.

\section{RESUMEN}

En el presente estudio se determinó el efecto de las asociaciones anestésicas atropina IM (0,05 mg/kg)-tiletamina/zolazepam EV (4 mg/kg total $) \mathrm{y}$ atropina IM $(0,05 \mathrm{mg} / \mathrm{kg}$-ketamina EV $(5 \mathrm{mg} / \mathrm{kg}$ total $) /$ diazepam $(0,5 \mathrm{mg} / \mathrm{kg})$ sobre la respuesta fisiológica, anestésica y bioquímica de emúes adultos. Los ejemplares ( $\mathrm{n}=7$ por grupo) fueron asignados al azar a dos tratamientos anestésicos. La frecuencia cardiaca y la temperatura corporal de los emúes mostraron un incremento al inicio del tratamiento experimental (entre 5-10 $\mathrm{min}, \mathrm{P}=0,001$ ). Sin embargo, la frecuencia respiratoria y pulso disminuyeron (entre 5-15 $\mathrm{min}, \mathrm{P}=0,003$ ). Estos patrones fueron detectados para ambas asociaciones anestésicas. Por otra parte, la inducción anestésica y el tiempo de recuperación anestésica no fueron afectados por los tratamientos $(\mathrm{P}=0,12$ y $\mathrm{P}=0,13$ respectivamente). Los emúes tratados con tiletamina mostraron un mayor tiempo de anestesia quirúrgica, comparados con los emúes tratados con ketamina $(\mathrm{P}=0,012)$. En el caso de A.S.T. y glucosa, ambas variables presentaron un incremento a las $24 \mathrm{~h}$ de la aplicación del tratamiento anestésico, resultando los niveles de glucosa más altos en emúes tratados con ketamina ( $\mathrm{P}=0,006$ y $\mathrm{P}=0,008$ respectivamente). Finalmente, la hemoglobina, proteínas totales y ácido úrico no presentaron diferencias significativas entre tratamientos $(\mathrm{P}=0,99, \mathrm{P}=0,97$ y $\mathrm{P}=0,81$ respectivamente $)$. En conclusión, los dos protocolos anestésicos resultaron seguros y eficientes para la manipulación de los animales; sin embargo, el mayor tiempo de anestesia observado en animales tratados con tiletamina podría determinar la preferencia por esta asociación anestésica.

\section{AGRADECIMIENTOS}

R. Pulgar agradece al proyecto interno de investigación y creación, Universal Santo Tomás: "Evaluación de ocho asociaciones anestésicas inyectables en emúes adultos".

J. Pulgar agradece al Proyecto DI0508, Universidad Andrés Bello.

\section{REFERENCIAS}

Adams HR. 1995. Cholinergic Pharmacology: Autonomic Drugs. In: Adams HR (ed). Veterinary Pharmacology and Therapeutics. $7^{\text {th }}$ ed. Iowa State University Press, Ames, Iowa, USA, Pp, 127-130. 
Alves GES, SM Hartsfield, GL Caroll, D Santos, S Zhang, RT Solis, A Baumñer, R Santos. 2003. Use of propofol, isoflurane and morphine for prolonged general anaesthesia in calves. Arq Bras Med Vet Zootec 55, 411-420.

Bruce X. 1998. Estudio preliminar de los efectos anestesiológicos de la asociación anestésica Tiletamina Zolazepam en caninos. Memoria de título, Escuela de Medicina Veterinaria, Universidad Santo Tomás, Santiago, Chile.

Ciboto R, SR Cortopassi, MA Lopes, RC Carvalho, CG Baitelo. 2006. Comparison of chemical restraint techniques in ostrich (Struthio camelus). Braz J Poult Sci 8, 119-123.

Cornick JL, J Jensen. 1992. Anesthetic management of ostriches. J Am Vet Assoc 200, 1661-1666.

Cornick-Seahorn JL. 1996. Anesthesiology of Ratites. In: Tully TN, Shane SM (eds). Management, medicine, and surgery. Krieger Publishing Company. Malabar, Florida USA, Pp 79-94.

Curro TG. 1998. Anesthesia of pet birds. Seminars in Avian and Exotic Pet Medicine 7, 10-21.

De Lucas JJ, C Rodriguez, M Marin, F González, C Ballesteros, MI San Andrés. 2007. Pharmacokinetics of intramuscular ketamine in young Ostriches premedicated with Romifidine. J Vet Med A 54, 48-50.

Fudge A. 1996. Clinical Hematology and Chemistry of Ratites. In: Tully $\mathrm{T}$, Shane S (eds). Management, medicine, and surgery. Krieger Publishing Company, Malabar, Florida, USA, Pp 105-113.

Grubb B, DD Jorgensen, M Conner. 1983. Cardiovascular changes in the exercising emu. J Exp Biol 104, 193-201.

Gunkel C, M Lafortune 2005. Current techniques in avian anesthesia. Seminars in Avian and Exotic Pet Medicine 14, 263-276.

Guyton A, J Hall. 1996. Distensibilidad vascular y funciones de los sistemas arterial y venoso. En: Guyton A, Hall J (ed). Tratado de Fisiología Medica. $10^{\mathrm{a}}$ ed. McGraw-Hill Interamericana, Madrid, España, Pp 185-195.

Johnson DC. 1996. Exotic companion medicine hand book for veterinarians, $1^{\text {st }}$ ed. Washington D.C., USA.

Kohrs R, ME Durieux. 1998. Ketamine: teaching an old drug new tricks. Anesth Analg 87, 1186-1193.

Laredo F. 2001. La preanestesia: analgesia, inmovilización farmacológica, tranquilización y ansiólisis. Consulta Difusión Veterinaria 77, 37-50.

Lin H, PG Todhunter, TA Power. 1997. Use of xylazine, butorphanol, tiletamine-zolazepam, and isoflurane for induction and maintenance of anaesthesia in Ratites. J Am Vet Med Assoc 210, 244-248.

Lopez-Timoneda F, MC. Gasco. 1993. Anestésicos generales. Hipnóticos. En: Velasco A, Fernández P, Serrano L, Velásquez JS (ed). Farmacología. 16 a ed. Madrid, España, Pp 395-415.

Lumeij JT. 1997. Avian Clinical Biochemistry. In: Kaneko J, Remple JD, Redig PT (eds). Clinical Biochemistry of Domestic Animals. Academic Press, San Diego, USA, Pp 857-879.
Miller RD. 2001 Anesthesia. $5^{\text {th }}$ ed. New York, Churchill Livingstone, Philadelphia, USA.

Mostachio GQ, LD de-Oliveira, AC Carcioli, WRR Vicente. 2008. The effects of anesthesia with a combination of intramuscular xylazinediazepam-ketamine on heart rate, respiratory rate and cloacal temperature in roosters. Veterinary Anesthesia and Analgesia 35, 232-236.

Muir WW, JA Hubbell, RT Skada. 2001. Manual de anestesia veterinaria. $2^{\mathrm{a}}$ ed. Mosby, Madrid, España.

Nevarez JG. 2005. Monitoring During Avian and Exotic Pet Anesthesia. Seminars in Avian and Exotic Pet Medicine 14, 277-283.

Paddleford RR. 2001. Manual de Anestesia de Pequeños Animales. $2^{\mathrm{a}} \mathrm{ed}$. Intermédica, Buenos Aires, Argentina, Pp 423.

Pereira J, A Barreiro, C Vásquez. 1996. Anestesia general. En: Gonzalo JM (ed). Cirugía veterinaria. McGraw-Hill Interamericana, Madrid, España, Pp 493-502.

Perrins C, A Middleton. 1990. Enciclopedia Ilustrada de las Aves. $1^{\mathrm{a}} \mathrm{ed}$. Plaza y Janés, Barcelona, España.

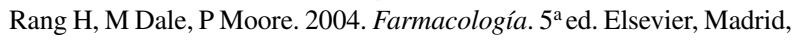
España.

Ritchie M. 1994. Avian Medicine, Principles and Application. $2^{\text {nd }}$ ed. California, USA.

Silva de Carvalho H, R Ciboto, C Grinaboldi, RA Dias, SR Gaido. 2007. Anatomia do sistema porta renal e suas implicações no emprego de agentes anestésicos na contenção de avestruzes (Struthio camelus). Ciência Rural 37, 1688-1694.

Smith DJ, AJ Azzaro, SB Zaldivar, S Palmer, HS Lee. 1981. Properties of the optical isomers and metabolites of ketamine on the high affinity transport and catabolism of monoamines. Neuropharmacology 20, 391-396.

StatSoft, INC 1995. STATISTICA for Windows (Computer Program Manual). Tulsa, USA.

Stewart JS. 1989. Husbandry, Medical and surgical management of Ratites: Part II. Proc Am Assoc Zoo Vet, 119-122.

Stewart J. 1994. Ratites. In: Harrison GJ, Harrison LR (eds). Avian Medicine, Principles and Application. Lake Woth, Florida, USA, Pp 184.

Sumano H, L Ocampo. 1997. Farmacología veterinaria. $2^{\mathrm{a}}$ ed. McGrawHill Interamericana, México DF, México.

Tully TN, SM Shane. 1996. Ratite Management, Medicine, and Surgery. Krieger Publishing Company. Malabar, Florida, USA, Pp 105-113.

Van Heerden J, RH Keffen. 1991. A preliminary investigation into the inmobilizing potential of a tiletamine/zolazepam mixture, metomidate, a metomidate and azaperone combination and medetomidine in ostriches (Struthio camelus). J S Afr Vet Assoc 62, 114-117.

Zar J. 1996. Biostatistical Analysis. Prentice-Hall, Englewood Cliffs, New Jersey, USA. 